\title{
Fatores Associados aos Problemas de Saúde Mental em Adolescentes ${ }^{1}$
}

\author{
Joviana Q. Avanci ${ }^{2}$ \\ Simone G. Assis \\ Raquel V. C.Oliveira \\ Renata M. Ferreira \\ Renata P. Pesce \\ Centro Latino-Americano de Estudos de Violência e \\ Saúde/Fundação Oswaldo Cruz.
}

\begin{abstract}
RESUMO - O objetivo do artigo foi investigar os problemas de saúde mental de adolescentes escolares e identificar alguns aspectos individuais, sociais e familiares associados ao seu desenvolvimento. Baseia-se num inquérito epidemiológico com 1.923 alunos de $7^{\mathrm{a}} / 8^{\mathrm{a}}$ séries e de $1^{\circ} / 2^{\circ}$ anos de escolas públicas e privadas do município de São Gonçalo, RJ. Para aferir os transtornos psiquiátricos menores utilizou-se a escala Self-Reported Questionnarie. Foram avaliadas questões relativas aos aspectos individuais, familiares e sociais. Utilizou-se a Regressão Logística Simples, tendo a razão de chances como medida para interpretação dos resultados. Constatou-se que violência psicológica, eventos difíceis do relacionamento familiar, auto-estima, satisfação com a vida, sexo e competência na escola se mantiveram no modelo final, indicando seu potencial em comprometer a saúde mental. A apresentação dos dados pode subsidiar o desenvolvimento de políticas públicas para o atendimento do adolescente, especialmente em termos preventivo e de promoção à saúde na área de saúde mental.
\end{abstract}

Palavras-chave: saúde mental; adolescente; problemas psiquiátricos menores.

\section{Associated Factors with Mental Health Problems in Adolescents}

\begin{abstract}
This article aims at investigating the mental health problems of school adolescents and identifying individual, social and family aspects associated with its development. It is based on an epidemical survey with 1923 students of the 7th and 8th grades of Elementary School and 1st and 2nd years of High School of public and private schools of municipality of São Gonçalo, RJ. The scale Self-Reported Questionnaire was used to evaluate Minor Psychiatric Morbidity. Individual, social and family aspects were evaluated. Simple Logistic Regression was utilized and the odds ratio was the measure to interpret the results. Psychological violence, difficult family life events, self-esteem, satisfaction with life, sex and school competence were maintained in the final model, indicating their potential to compromise mental health. The presentation of the data can subsidize the development of public policies for the attendance of the adolescent, especially in the preventive level and in the promotion of health in the mental health area.
\end{abstract}

Key words: mental health; adolescent; minor psychiatric morbidity.

A adolescência é tradicionalmente conhecida como uma fase de instabilidade emocional e de explosão de crescimento, com mudanças físicas e psicossociais. Período de transição e de nova relação com o mundo adulto, o adolescente encontrase diante de conflitos pessoais e familiares, questionamentos e ambivalências, podendo confundir a si mesmo e aos que o cerca sobre os limites de "normalidade" (Feijó \& Chaves, 2002).

É nesse período da vida que os momentos naturalmente depressivos e conflitantes são característicos, a ponto de alguns autores questionarem se essa fase da vida seria um

1 A pesquisa recebeu suporte do Fundo das Nações Unidas para a Infância (UNICEF) e também contou com bolsistas do Programa PIBIC do $\mathrm{CNPq} /$ Fiocruz.

2 Endereço: Avenida Brasil, 4036, sala 700, Manguinhos, Rio de Janeiro, RJ, Brasil 21040-631.E-mail: joviana@claves.fiocruz.br processo de luto ou depressão. Esse processo se daria pelo luto do corpo infantil, pela perda dos pais da infância, pela perda da identidade infantil e pela própria afirmação do adolescente no mundo adulto (Aberastury, 1983; Sukiennik $\&$ Salle, 2002). Paradoxal e complementarmente, a fase da adolescência tem importância vital para a estruturação de personalidade do indivíduo, além de ser uma época da vida em que os melhores índices de saúde, vitalidade e criatividade são vividos, permitindo que o adolescente responsabilize-se pelas tarefas da vida adulta e se lance em novos sonhos e perspectivas.

Pelas inúmeras confluências e fragilidades psicossociais que cercam a fase da adolescência, fatores relacionados à saúde mental se sobressaem nas análises desse período da vida. Diversos estudos discutem o risco elevado do suicídio e da depressão na adolescência, chegando a $20 \%$ a prevalência de depressão nessa faixa etária em alguns países (Lewinsohn, Hops, Roberts, Seeley \& Andrews, 1993; Maj \& Sartorius, 
2005). Em relação ao estado de saúde mental de maneira geral, os problemas podem variar de $3,5 \%$ a $49 \%$ em adolescentes ingleses e italianos, respectivamente, com instrumental diagnóstico diferenciado (Banks, 1983; Seva, Sarasola, Merino \& Magallon, 1992).

No Brasil, um inquérito epidemiológico detectou que $14,3 \%$ de adolescentes escolares de um município do Rio de Janeiro já pensaram em acabar com a sua própria vida (Assis, Pesce \& Avanci, 2006), sendo escassos os trabalhos populacionais ou com amostras representativas que investiguem o estado geral da saúde mental nas populações, em especial de adolescentes. Na infância, Vitolo, Fleitlich-Bilyk, Goodman e Bordin (2005) mostram que 35,2\% de crianças escolares de 7 a 11 anos de um município de São Paulo denotam problemas de saúde mental. Na fase adulta, estudos conduzidos em clientelas de serviços de saúde de duas capitais do Sudeste e Sul do país constataram que cerca de $50 \%$ dos pacientes atendidos apresentavam algum transtorno mental comum (Busnello, Lima \& Bertolote, 1983; Mari, 1987). E, numa amostra não enviesada, Lopes, Faerstein e Chor (2003) estimam em $29 \%$ os transtornos mentais menores em funcionários públicos técnicoadministrativos de uma universidade do Rio de Janeiro.

Neste trabalho, assim como em outros estudos (Bener \& Tewfik (2006); Loyaza \& cols., 2001; Vitolo \& cols, 2005), utiliza-se a categoria dos transtornos psiquiátricos menores (TPM) para o estudo dos problemas de saúde mental. Num limiar entre a saúde e a doença, esse construto caracteriza-se fundamentalmente por uma experiência subjetiva de mal-estar inespecífico, com repercussões fisiológicas e psicológicas. Dependendo da intensidade e da gravidade, pode acarretar limitações ao dia-a-dia e prejuízos importantes na qualidade de vida, transformando-se em um grave problema de saúde e, inclusive, elevando as taxas de mortalidade (Coutinho, 1995; Huppert \& Whittington, 1995). A ocorrência de problemas mentais nas fases da infância e adolescência é ainda mais grave, visto ser consenso a alta probabilidade de recorrência de morbidades psiquiátricas em fases da vida mais tardias (Harrington, Fudge, Rutter, Pickels \& Hill, 1990; Kovacs \& cols, 1984).

Os TPM na adolescência são sabidamente associados aos graves problemas de saúde pública, entre eles estão as desordens alimentares, o uso de drogas e os comportamentos anti-sociais, sem levar em conta os problemas na esfera cognitiva e da sexualidade, o que revela a premência do tema para a saúde infanto-juvenil (Falcone, Mäder, Nascimento, Santos \& Nóbrega, 2005; Feijó \& Oliveira, 2001; Loyasa \& cols, 2001).

Vários fatores tornam a criança e o adolescente mais vulneráveis a sofrerem problemas na área de saúde mental: a) os individuais (sexo; idade; características psicológicas como auto-estima, autoconfiança e determinação), b) os familiares (história de problemas de saúde mental, especialmente materna; problemas de álcool/drogas; violência física, psicológica e sexual; violência entre os pais; perdas por morte; separação dos pais), c) os socioculturais (pobreza, violência no contexto social, apoio/suporte social) e d) os biológicos (Assis \& cols, 2006; Kestilä \& cols, 2006; Manninen, Heliövaara, Riihimäki \& Mäkelä, 1997; Vitolo \& cols, 2005). O grande desafio é entender como esses fatores articulam-se entre si e engendram-se no comportamento e na saúde humana.
Embora em termo mundial, um dos principais objetivos da epidemiologia aplicada à Psiquiatria Infantil tem sido determinar a distribuição da psicopatologia infanto-juvenil, aumentando o conhecimento sobre as características, a etiologia e os fatores de risco dos problemas de saúde mental, no Brasil, são escassos os trabalhos que se debruçam sobre essa temática (Bird, 1996; Feijó, Saueressig, Salazar \& Chaves, 1997; Lopes \& cols., 2003; Loyaza \& cols, 2001). Essa lacuna dificulta o apropriado planejamento de estratégias preventivas na área da saúde mental e conseqüentemente o delineamento de políticas públicas juvenis.

Considera-se, portanto, que a relevância acadêmica do tema e a reduzida produção empírica nacional a respeito tornam pertinente a investigação dos fatores associativos que se destacam aos problemas de saúde mental de adolescentes no contexto urbano brasileiro. Por um lado, a identificação de determinantes individuais, sociais e familiares em regiões metropolitanas e de grande vulnerabilidade social adquire importância estratégica, já que se trata da realidade vivida por grande parte da população brasileira. Por outro lado, além de custos elevados, as características da vida urbana atuais impõem dificuldades operacionais para a realização de pesquisas de campo na população geral. A abordagem de populações escolares no ambiente de ensino constitui uma alternativa bastante plausível, seja pelo alcance de maior tamanho amostral, possibilitando maior poder estatístico nas análises realizadas, seja pela relativa facilidade de acesso, auxiliando a operacionalização da coleta dos dados; ou mesmo por ser um local privilegiado, no qual boa parte dos adolescentes freqüenta, coadunando experiências e realidades distintas. Essas vantagens facilitam o alcance dos objetivos propostos (Lopes \& cols., 2003).

Este trabalho objetiva investigar sobre os problemas de saúde mental de adolescentes escolares de um município do Rio de Janeiro, assim como identificar alguns aspectos individuais, sociais e familiares associados ao desenvolvimento dessa problemática na população adolescente do referido município.

\section{Método}

Os dados inéditos analisados neste artigo são originários de um inquérito epidemiológico desenvolvido em 2003, no município de São Gonçalo/RJ. Esse município integra a Região Metropolitana do Estado do Rio de Janeiro, sendo o segundo maior município do estado em termos populacionais e o $15^{\circ}$ do país.

Participaram 1.923 alunos entre 10 e 19 anos das $7^{\mathrm{a}}$ e $8^{\mathrm{a}}$ séries do Ensino Fundamental $(51,8 \%)$ e $1^{\circ}$ e $2^{\circ}$ anos do Ensino Médio (48,2\%), do turno diurno, matriculados na rede pública e privada de São Gonçalo. A amostra constituiu de 35,7\% de alunos de 10-14 anos e $63.4 \%$ de $15-19$ anos; $55,7 \%$ do sexo feminino e $44,3 \%$ do masculino; $50,2 \%$ se autodenominavam negros e pardos, $43,4 \%$ brancos e $6,4 \%$ consideravam a cor de pele amarela/indígena. Quanto à religião, 76\% afirmam serem filiados a cultos religiosos enquanto $24 \%$ não seguem qualquer prática religiosa.

A seleção dos alunos foi realizada mediante amostragem por conglomerado em dois estágios: escolas e turmas. Participaram todos os alunos que estavam presentes no dia da aplicação nas 68 turmas e 38 escolas amostradas. Dos 1.923 
entrevistados, 997 foram da rede pública e 926 da rede particular. A pesquisa foi autorizada pela Secretaria Municipal de Educação do Município, aprovada pelo Comitê de Ética em Pesquisa da Escola Nacional de Saúde Pública/FIOCRUZ, e os envolvidos (adolescentes e direção das escolas participantes) assinaram consentimentos livre-esclarecido, conforme preconiza as normas éticas de pesquisas envolvendo seres humanos.

\section{Instrumentos}

Foi utilizado um questionário multidimensional, anônimo e autopreenchível aplicado nas turmas com o apoio de equipe treinada. Entre outras questões, o questionário abordou aspectos individuais, familiares e sociais: a experiência de violência física (Straus, 1979); de violência na escola e na comunidade (Kahn \& cols, 1999); de violência sexual (Sanders \& Becker-Lausen, 1995); violência psicológica (Pitzner \& Drummond, 1997); apoio emocional (Sherbourne \& Stewart citados por Chor, Faerstein, Alves \& Lopes, 2001); auto-estima (Rosenberg, 1989); satisfação com a vida (Diener, Emmons, Larsen \& Grifin, 1985); coping - formas de resolução de problemas (Kavek \& Seiffge-Krenk, 1996); jovem transgressor (Kahn \& cols, 1999); resiliência (Wagnild \& Young, 1993); uso de drogas (CEBRID, 1997) e eventos de vida adversos (Pitzner \& Drummond, 1997; Trombeta \& Guzzo, 2002). Todas as escalas foram testadas em estudos pilotos, apresentando índices psicométricos adequados para aplicação (Assis \& cols, 2006).

Para aferir a presença de TPM utilizou-se a escala SRQ20 (Self-Reported Questionnarie) desenvolvida por Harding, Arango, Baltazar, Climent e Ibrahim (1980), que rastreia sintomas: 1) psicossomáticos (dores de cabeça, má digestão e sensações desagradáveis no estômago; 2) depressivos (tristeza, choro freqüente, falta de apetite, perda de interesse, sentimento de inutilidade e sem valor, idéia de acabar com a vida, cansaço, dificuldade de pensar com clareza e de realizar com satisfação atividades diárias); 3) ansiosos (dormir mal, assustar-se com facilidade, tremores na mão, nervosismo/ tensão/agitação, dificuldade para tomar decisões, dificuldades na escola - item adaptado do original o qual afere dificuldades no trabalho).

A versão brasileira da SRQ-20 foi desenvolvida por Busnello e cols. (1983) e validada por Mari e Williams (1986), sendo composta por 20 itens (sim/não). Foram considerados casos aqueles do sexo masculino que foram positivos em sete ou mais itens, e oito ou mais para os do sexo feminino, conforme proposto pelo autor original. Esse instrumento já é bem estabelecido e amplamente utilizado em trabalhos internacionais e nacionais, inclusive com amostra adolescente (Bener \& Tewfik, 2006; Feijó \& cols, 1997; Oehlsschlaeger, Pinheiro, Horta, Gelatti \& Sant'Tana, 2004). A avaliação psicométrica, efetuada a partir de dados de estudo piloto (210 adolescentes) e feita por meio de confiabilidade teste-reteste, apresentou ICC (Intraclass Correlation Coeficient) de 0,85 e alpha de Cronbach de 0,75 . A validade de construto foi obtida pela correlação inversa com apoio social, auto-estima e satisfação na vida e correlação positiva com experiência de violência física e psicológica (Assis \& cols, 2006).

\section{Análise dos dados}

Em uma primeira análise, a relação entre TPM e as questões individuais, familiares e sociais foram examinadas pelo critério da significância estatística referente a 5\% pelo teste qui-quadrado. Posteriormente, a associação entre as variáveis foi estudada pelo método de regressão logística univariada para cada uma das 35 variáveis explicativas do modelo inicial, o que resultou em apenas três variáveis nãosignificativas (religião, resiliência e faixa etária - teste de Wald) (Tabela 1).

Com as 32 variáveis resultantes foi realizada análise multivariada, testadas segundo níveis de abordagem teoricamente correlacionados aos TPM (Victora, Huttly, Fuchs \& Olinto, 1997): distal (variáveis do meio mais externo ao indivíduo), familiar (questões relacionadas ao universo familiar) e individual (variáveis referentes a características próprias do indivíduo). Essa análise foi efetuada em três passos, utilizando como regra de passagem para o próximo nível - 5\% teste de Wald tipo III: A) Passo 1: modelo logístico para as seis variáveis do nível distal; B) Passo 2: modelo incluindo as variáveis significativas no nível distal e todas as do nível familiar; C) Passo 3: modelo para as variáveis que permanecem no modelo anterior mais as do nível individual.

Para a composição do modelo final foi testado um modelo alternativo com as sete variáveis oriundas do modelo resultante do passo 3 e/ou aquelas que se mostraram significativas nas 35 análises univariadas, mantendo-se a lógica de inserção de variáveis em blocos, visando reduzir o confundimento. $\mathrm{O}$ modelo final ficou composto por seis variáveis. Realizou-se também um estudo das interações (duas a duas) sem apresentar significância estatística. O efeito das questões individuais, familiares e distais no status de caso dos TPM foi expresso por intermédio das odds ratios (OR) e seus respectivos intervalos de confiança (IC 95\%). As análises foram realizadas usando-se o programa estatístico SAS V8.0.

\section{Resultados}

A prevalência de transtornos psiquiátricos menores, segundo o ponto de corte adotado, foi de $29,4 \%$ dos adolescentes (63,4\% do sexo feminino e $36,6 \%$ do masculino), não havendo associação estatisticamente significativa entre os TPM e a natureza da escola (pública e particular) e nem com as séries escolares $\left(7^{\mathrm{a}}\right.$ e $8^{\mathrm{a}} / 1^{\mathrm{o}}$ e $\left.2^{\mathrm{o}}\right)$.

No primeiro teste da análise multivariada, três variáveis do nível distal mostraram associação estatisticamente significativa: relacionamento com professores, com amigos e eventos adversos com amigos e namorado. Essas variáveis permanecem significativas no modelo posterior e mais todas as do nível familiar - relacionamento com professor, eventos adversos no relacionamento familiar, com amigos e namorado, violência psicológica. $\mathrm{O}$ terceiro modelo multivariado englobou as quatro variáveis do modelo anterior mais todas do nível individual, resultando em sete variáveis: eventos adversos no relacionamento familiar, com amigos e namorado, violência psicológica, auto-estima, satisfação com a vida, sexo e participação em sala de aula.

Refinando ainda mais, obteve-se um modelo final com seis variáveis, o qual se excluiu apenas eventos adversos 
com amigos e namorados. A Tabela 2 apresenta as variáveis que se mantiveram no modelo final da regressão logística, de acordo com os níveis familiar e individual. As variáveis do nível distal não permaneceram, o que pode indicar fraco poder de associação com os TPM de adolescentes.

$\mathrm{Na}$ esfera familiar, a violência psicológica é o fator que mais se associa. Aqueles que viveram mais humilhações, em que alguém significativo para o adolescente reduziu suas qualidades, capacidades, desejos e emoções, além de cobrá-lo excessivamente, apresentam 4,17 vezes mais chance de apresentar transtornos psiquiátricos menores em relação àqueles que não sofrem essa forma de violência. Entre os que sofrem violência psicológica em uma forma menos severa, observa-se 1,65 vezes mais chance de apresentar os transtornos estudados do que entre os que não sofrem essa violência emocional.

Os adolescentes que passaram por muitas dificuldades no relacionamento familiar, entre elas as discussões entre familiares, a separação dos pais, novo casamento de pai e/ ou mãe e o incômodo com o nascimento de um novo irmão, apresentam 3,15 vezes mais chance de TPM que aqueles que tiveram poucos eventos difíceis no universo familiar.

Nos fatores do nível individual, ressaltam-se os de proteção à saúde mental: auto-estima, satisfação com a vida e

Tabela 1. Teste de significância nos modelos univariados segundo Variáveis Distais, familaires e Individuais e os Transtornos Psiquiátricos Menores em Adolescentes Escolares de São Gonçalo/RJ.

\begin{tabular}{|c|c|c|c|c|c|}
\hline NÍVEL DISTAL & Teste de Wald & NÍVEL FAMILIAR & Teste de Wald & NÍVEL NDIVIDUAL & Teste de Wald \\
\hline Relacionamento com amigos & 0,0008 & Supervisão Familiar & 0,0002 & Auto-estima & 0,0000 \\
\hline Relacionamento com professores & 0,0000 & Apoio Emocional & 0,0000 & Autodeterminação & 0,0003 \\
\hline Relacionamento c/ outra classe social & 0,0022 & Estrutura Familiar & 0,0022 & Auto-confiança & 0,0000 \\
\hline Eventos de Vida-Amigos e namorado & 0,0000 & Relacionamento com pai & 0,0000 & Sexo & 0,0000 \\
\hline Violência na escola & 0,0000 & Relacionamento com mãe & 0,0000 & Religião & 0,2925 \\
\hline \multirow[t]{10}{*}{ Violência na localidade } & 0,0000 & Relacionamento com irmãos & 0,0000 & Satisfação de vida & 0,0000 \\
\hline & & Violência severa pai na vida & 0,0000 & $\begin{array}{c}\text { Desempenho } \\
\text { matemática }\end{array}$ & 0,0090 \\
\hline & & Violência severa mãe na vida & 0,0000 & $\begin{array}{l}\text { Participação sala de } \\
\text { aula }\end{array}$ & 0,0000 \\
\hline & & Violência sexual na família & 0,0006 & Faixa Etária & 0,2813 \\
\hline & & Violência psicológica & 0,0000 & Coping Interno & 0,0078 \\
\hline & & Violência entre pais & 0,0000 & Coping Evitação & 0,0000 \\
\hline & & Violência entre irmãos & 0,0000 & Resiliência & 0,0847 \\
\hline & & Eventos médicos & 0,0000 & Jovem Transgressor & 0,0000 \\
\hline & & Eventos sócio-econômicos & 0,0000 & Uso de drogas & 0,0000 \\
\hline & & $\begin{array}{c}\text { Eventos relacionamento } \\
\text { familiar }\end{array}$ & 0,0000 & & \\
\hline
\end{tabular}

Tabela 2. Modelo logístico multivariado explicativo da presença de transtornos psiquiátricos menores em adolescentes escolares de São Gonçalo/RJ. $\left.{ }^{*}\right)$

\begin{tabular}{|c|c|c|c|c|c|}
\hline \multicolumn{2}{|c|}{$\begin{array}{l}\text { Variáveis explicativas da presença de transtornos } \\
\text { psiquiátricos menores }(N=1322)\end{array}$} & Razões brutas & $\begin{array}{l}\text { Intervalo de } \\
\text { confiança }\end{array}$ & Razão de chance & $\begin{array}{c}\text { Intervalo de } \\
\text { confiança }\end{array}$ \\
\hline \multicolumn{6}{|l|}{ Familiar } \\
\hline \multirow{3}{*}{ Violência psicológica } & Alta & 7,71 & $5,30-11,20$ & 4,17 & $2,68-6,48$ \\
\hline & Média & 2,11 & $1,43-3,12$ & 1,65 & $1,06-2,56$ \\
\hline & Ausente & 1,00 & - & 1,00 & - \\
\hline \multirow{3}{*}{$\begin{array}{l}\text { Eventos de vida } \\
\text { Relacionamento familiar }\end{array}$} & Alta & 4,88 & $3,29-7,23$ & 3,15 & $1,75-5,66$ \\
\hline & Média & 1,91 & $1,45-2,51$ & 1,30 & $0,88-1,92$ \\
\hline & Ausente & 1,00 & - & 1,00 & - \\
\hline \multicolumn{6}{|l|}{ Individual } \\
\hline \multirow{3}{*}{ Auto-estima } & Baixa & 8,32 & $6,02-11,50$ & 6,95 & $4,56-10,61$ \\
\hline & Moderada & 2,87 & $1,99-4,14$ & 3,05 & $1,90-4,90$ \\
\hline & Alta & 1,00 & - & 1,00 & - \\
\hline \multirow{2}{*}{ Satisfação com a vida } & Não & 3,85 & $2,87-5,17$ & 2,22 & $1,45-3,39$ \\
\hline & Sim & 1,00 & - & 1,00 & - \\
\hline \multirow{2}{*}{ Sexo } & Masculino & 0,61 & $0,49-0,77$ & 0,51 & $0,35-0,73$ \\
\hline & Feminino & 1,00 & - & 1,00 & - \\
\hline \multirow{4}{*}{$\begin{array}{l}\text { Competência: Participação } \\
\text { em sala de aula }\end{array}$} & Fraca & 2,63 & $1,73-4,00$ & 2,45 & $1,37-4,37$ \\
\hline & Regular & 1,12 & $0,83-1,53$ & 0,93 & $0,60-1,47$ \\
\hline & Boa & $\mathbf{0 , 8 3}$ & $0,62-1,10$ & 0,79 & $0,52-1,20$ \\
\hline & Ótima & 1,00 & - & 1,00 & - \\
\hline
\end{tabular}

${ }^{(*)}$ Em negrito os resultados que obtiveram significância estatística. 
competência na escola. Jovens com baixa auto-estima têm quase sete vezes mais chance de apresentar TPM que os de elevada alta-estima. Os que não são satisfeitos com suas vidas apresentam 2,22 vezes mais os transtornos investigados em relação àqueles que o são.

Tradicionalmente, o sexo feminino se configura como um risco a desordem psiquiátrica. Coadunando-se com isso, meninos têm menores chances de ter problemas na saúde mental se comparado às meninas. Quanto à competência escolar, representada pela participação em sala de aula, aqueles com fraca participação em sala de aula apresentam 2,45 vezes mais chances de apresentar TPM do que os que dizem ter uma participação mais ativa em sala de aula.

\section{Discussão}

Os resultados deste trabalho são representativos de toda população escolar das séries investigadas no município estudado. Comparações com realidades semelhantes podem ser compatíveis, no entanto, é importante um conhecimento mais apurado da população a que se pretende pesquisar, pois características locais podem induzir resultados díspares.

Os achados destacam resultados encontrados em estudos prévios realizados no Brasil e em outros países, a exemplo da vitimização de violência (Mazza \& Reynolds, 1999; Vitolo \& cols., 2005). Sofrer violência psicológica foi o fator mais associado aos TPM, o que se revela ainda mais preocupante mediante a naturalização dessa forma de se relacionar na sociedade, principalmente na educação de crianças e adolescentes ( $48 \%$ dos adolescentes estudados relatam sofrer essa forma de violência de pessoas significativas em suas vidas). Isso interfere no desenvolvimento de processos psicológicos básicos como auto-estima, autodeterminação e capacidade de resiliência e perpassa outras formas de vitimização, ocorrida em casa, na escola ou na comunidade, acarretando riscos ao desenvolvimento comportamental, emocional, social, cognitivo e físico de crianças e adolescentes até a vida adulta (Assis \& Avanci, 2004; Assis \& cols., 2006; Garbarino, Guttmann $\&$ Seeley, 1986).

É consensual que o relacionamento familiar, independente da cultura em que a criança ou o adolescente viva, exerce fundamental importância à estruturação psíquica do indivíduo. Sobre isso, Harrington (2005) comenta que crianças hospitalizadas por depressão, quando retornam à família com altos índices de crítica e discórdia, têm resultados muito piores do que aquelas que retornam a ambientes mais harmoniosos. Sobre o divórcio, Garmezy e Rutter (1988) comentam que relações familiares anteriores e posteriores a esse evento são determinantes às conseqüências no desenvolvimento de problemas mentais de crianças e adolescentes. São as inconsistências, as dificuldades de afeto, de relacionamento e a falta de controle dos filhos, comuns nas separações dos pais, que realmente interferem na saúde mental infanto-juvenil.

Ser do sexo feminino, ter baixa auto-estima, insatisfação pela vida e pouca participação acadêmica são questões tradicionais nos estudos sobre problemas de saúde mental, sendo inclusive considerados como fatores de risco aos TPM (Feijó \& cols, 1997; Miquelon, Vallerand, Grouzet \& Cardinal, 2005; Ustárroz, 1997). Além disso, alguns desses são sinais e sintomas importantes no diagnóstico psiquiátrico, reiterando a importância desses aspectos no estado de saúde mental das pessoas.

Causa estranheza a não permanência no modelo explicativo final dos TPM de questões que têm estado no cenário acadêmico mundial dos estudos da Psiquiatria Infanto-juvenil, como a violência física originada na família e na comunidade e as condições socioeconômicas precárias. Porém, aportes metodológicos diferenciados podem contribuir para a melhor elucidação desses resultados. Não obstante, numa análise mais simplificada, encontra-se associação entre essas questões e os transtornos psiquiátricos menores.

Aliás, as principais limitações deste estudo decorrem: 1) da utilização de dados seccionais, não permitindo investigar a possibilidade de causalidade reversa e 2) da abordagem de análise realizada pela regressão logística, que pode gerar confundimento pelo grande número de variáveis envolvidas. Quanto à primeira delimitação, Lopes e cols. (2003) colocam que distinguir fatores de risco de efeitos adversos constitui um dos grandes desafios da área e ressalta a necessidade do desenvolvimento de trabalhos longitudinais, especialmente da investigação de questões que podem mediar os TPM, funcionando como proteção ou buffer. Quanto à segunda, tentou-se minimizar esse problema pela análise em blocos (abordagens teóricas) e por meio das análises univariadas. Essa abordagem em blocos de análise está em sintonia com a proposta de desenvolvimento sugerida por Bronfenbrenner (1979), a qual examina a criança/adolescente num sistema ecológico, numa série de camadas ou círculos concêntricos, desde a interação familiar até a influência cultural.

Outra limitação refere-se ao fato do instrumental de aferição utilizado (SRQ-20) ser sensível a recentes mudanças no estado psicológico das pessoas, não permitindo a distinção daqueles com diagnóstico estabelecido. Lopes e cols. (2003) enfatizam que tal fato pode levar à inclusão de falsos positivos no estudo (indivíduos com transtornos transitórios), superestimando a prevalência de TPM nessa população. No entanto, o resultado de prevalência encontrado em outros trabalhos, variando de $20 \%$ a $30 \%$, sugere que a SRQ-20, com os pontos de corte adotados, é um instrumento adequado para a utilização no estudo dos TPM nessa população (Bener \& Tewfik, 2006; Oehlsschlaeger \& cols., 2004).

A abordagem metodológica utilizada deixa de lado alguns aspectos sobre o experienciar os transtornos psiquiátricos menores (TPM). Sabe-se que os problemas de saúde mental não têm manifestação única para todos os indivíduos dentro de uma mesma família, cultura ou período histórico (Brant \& Minayo, 2004). O que é sofrimento para um, não é, necessariamente, para outro, mesmo quando submetidos às mesmas condições ambientais adversas. Sabe-se também que a cultura exerce uma diferença na forma pela qual o malestar é expresso, por exemplo, sintomas somáticos são mais comuns na cultura oriental, ao passo que no Ocidente os sintomas afetivos e cognitivos são mais freqüentes, pela própria educação com que as crianças e adolescentes são criadas nas duas culturas (Stewart \& cols., 2002). Isso traz à tona a urgente necessidade de se investir em estudos com abordagens metodológicas variadas, especialmente qualitativas.

Certamente a apresentação dos dados pode subsidiar o desenvolvimento de políticas públicas para o atendimento do adolescente. $\mathrm{O}$ estudo dos fatores associados aos TPM 
na fase da adolescência ressalta a relevância do tema no debate de políticas públicas juvenis à área de saúde mental. Afirma também a necessidade de os profissionais de saúde e de educação estarem sensibilizados e preparados para lidarem com aspectos emocionais dos indivíduos, adotando estratégias preventivas e promotoras de saúde. Sem dúvida que uma abordagem dessa natureza pode assumir um poder multiplicador, prevenindo também outros agravos à saúde e minimizando difíceis e graves conseqüências à saúde até a vida adulta.

\section{Referências}

Aberastury, A. (1983). Adolescência. Porto Alegre: Artmed.

Assis, S. G. \& Avanci, J. Q. (2004). Labirinto de espelhos: Formação da auto-estima na infância e na adolescência. Rio de Janeiro: Fiocruz.

Assis, S. G., Pesce, R. P. \& Avanci, J. Q. (2006). Resiliência. Enfatizando a proteção dos adolescentes. Porto Alegre: Artmed.

Banks, M. H. (1983). Validation of the general health questionnaire in a young community sample. Psychological Medicine, 13, 349-353.

Bener, A. \& Tewfik, I. (2006). Prevalence of overweight, obesity, and associated psychological problems in Qatari's female population. Obesity Reviews, 7, 139-145.

Bird, H. R. (1996). Epidemiology of childhood disorders in a crosscultural context. Journal of Child Psychology and Psychiatry, 37(1), 35-49.

Brant, L. C. \& Minayo, C. G. (2004). A transformação do sofrimento em adoecimento: do nascimento da clínica à psicodinâmica do trabalho. Ciência \& Saúde Coletiva, 9(1), 213-223.

Bronfenbrenner, U. (1979). The Ecology of Human Development. Cambridge: Harvard University Press.

Busnello, E. L., Lima, B. \& Bertolote, J. M. (1983). Aspectos interculturais de classificação e diagnóstico. Jornal Brasileiro de Psiquiatria, 32(4), 207-210.

CEBRID - Centro Brasileiro de Informações sobre Drogas Psicotrópicas (1997). IV Levantamento Sobre o Uso de Drogas entre Estudantes de $1^{\circ}$ e $2^{\circ}$ graus em 100 Capitais Brasileiras. São Paulo: CEBRID.

Chor, D., Faerstein, E., Alves, M. G. M. \& Lopes, C. S. (2001). Medidas de rede e apoio social no Estudo Pró-Saúde: pré-testes e estudo piloto. Cadernos de Saúde Pública, 17(4), 887-896.

Coutinho, E. S. F. (1995). Fatores Sócio-demográficos e Morbidade Psiquiátrica Menor: Homogeneidade e Heterogeneidade de Efeitos. Tese de Doutorado, Universidade Federal da Bahia, Salvador.

Diener, E, Emmons, R. A., Larsen, R. J. \& Grifin, S. (1985). The satisfaction with life scale. Journal of Personality Assessment, 49, 71-75.

Falcone, V. M., Mäder, C. V. de N., Nascimento, C. F. L., Santos, J. M. M. \& Nóbrega, F. J. (2005). Multiprofessional care and mental health in pregnant women. Revista de Saúde Pública, 39(4), 1-6.

Feijó, R. B. \& Chaves, M. L. F. (2002). Comportamento suicida. Em M. C. O. Costa \& R. P. de Souza (Orgs.), Adolescência (pp. 398-408). Porto Alegre: Artmed Editora.

Feijó, R. B. \& Oliveira, E. A. (2001). Comportamento de risco na adolescência. Jornal de Pediatria, 77(2), S125-S134.
Feijó, R. B., Saueressig, M., Salazar, C. \& Chaves, M. L. F. (1997). Mental Health Screening by self-report questionnaire among community adolescents in Southern Brazil. Journal of Adolescent Health, 20, 232-237.

Garbarino, J., Guttmann, E. \& Seeley, J. W. (1986). The Psychologically battered child. San Francisco: Jossey - Bass Publishers.

Garmezy, N. \& Rutter, M. (1988). Stress, Coping and Development in Children. New York: McGraw Hill.

Harding, T. W., Arango, M. V., Baltazar, J., Climent, C. E. \& Ibrahim, H. H. A., Ignacio L. L., Murthy, R. S. \& Wig, N. N. (1980). Mental disorders in primary health care: a study of their frequency and diagnosis in four developing countries. Psychological Medicine, 10, 231-241.

Harrington, R. C., Fudge, H., Rutter, M., Pickels, A. \& Hill, J. (1990). Adult outcomes of childhood and adolescents depression. Risk for antisocial disorders. Journal of the American Academy of Child \& Adolescent Psychiatry, 30, 434-439.

Harrington, R. (2005). Transtornos Depressivos em Crianças e Adolescentes: uma revisão. Em M. Maj \& N. Sartorius (Orgs.), Transtornos Depressivos (pp. 191-248). Porto Alegre: Artmed.

Huppert, F.A. \& Whittington, J.E. (1995). Symptoms of psychological distress predict 7-year morbidity. Psychological Medicine, 25(5), 1073-86.

Kahn, T., Bermergui, C., Yamada, E., Cardoso, F. C., Fernandes, F., Zacchi, J. M., Guimarães, L. \& Hasselman, M. E. (1999). O dia a dia nas escolas (violências auto-assumidas). Projeto de Pesquisa. São Paulo: Instituto Latino Americano das Nações Unidas para a Prevenção do Delito e Tratamento do Delinquente (ILANUD) e Instituto Sou da Paz.

Kavek, M. J. \& Seiffge-Krenk, I. (1996). The differentiation of Coping Traits in Adolescence. International Journal of Behavioral Development, 19(3), 651-668.

Kestilä, L., Koskinen, S., Martelin, T., Rahkonen, O., Pensola, T., Aro, H. \& Aromaa, A. (2006). Determinants of health in early adulthood: what is the role of parental education, childhood adversities and own education? The European Journal of Public Health, 16(3), 305-314.

Kovacs, M., Feinberg, T. L., Crouse-Novak, M., Paulauskas, S. L. Pollock, M. \& Finkelstein, R. (1984). Depressive disorders in childhood. II. A longitudinal prospective study of characteristics and recovery. Archives of General Psychiatry, 41, 643-649.

Lewinsohn, P. M., Hops, H., Roberts, R. E., Seeley, J. R. \& Andrews, J. A. (1993). Prevalence and Incidence of depression and other DSM-III-R disorders in high school students. Journal of Abnormal Psychology, 102, 133-144.

Lopes, C. S., Faerstein, E. \& Chor, D. (2003). Eventos de vida produtores de estresse e transtornos mentais comuns: resultados do Estudo Pró-Saúde. Cadernos de Saúde Pública, 19(6), 1713-1720.

Loyaza, M. P., Ponte, T. S., Carvalho, C. G.; Pedrotti, M. R., Nunes, P. V., Souza, C. M., Zanette, C. B. \& Chaves, M. L. F. (2001). Association between mental health screening by self-report questionnaire and insomnia in medical students. Arquivos de Neuro-Psiquiatria, 59(2-A), 180-185.

Maj, M. \& Sartorius, N. (2005). Transtornos Depressivos. Porto Alegre: Artmed. 
Manninem, P., Heliövaara, M., Riihimäki, H. \& Mäkelä, P. (1997). Does Psychological Distress predict disability? International Journal of Epidemiology, 26(5), 1063-1070.

Mari, J. J. (1987). Psychiatry Morbidity in three primary medical care clinics in the city of São Paulo. Issues on the mental health of the urban poor. Social Psychiatry, 22, 128-139.

Mari, J. J. \& Williams, P. (1986). A validity study of a psychiatric screening questionnaire (SRQ-20) in primary care in the city of São Paulo. British Journal of Psychiatry, 148, 23-26.

Mazza, J. J. \& Reynolds, W. M. (1999). Exposure to Violence in Young Inner-City Adolescents: Relationships with suicidal ideation, depression, and PTSD symptomatology. Journal of Abnormal Child Psychology, 27(3), 203-213.

Miquelon, P., Vallerand, R. J., Grouzet, F. M. E. \& Cardinal, G. (2005). Perfectionism, Academic Motivation, and Psychological Adjustment: An Integrative Model. Personality and Social Psychology Bulletin, 31(7), 913-924.

Oehlsschlaeger, M. H. K., Pinheiro, R. T., Horta, B., Gelatti, C. \& Sant'Tana, P. (2004). Prevalência e fatores associados ao sedentarismo em adolescentes de área urbana. Revista Saúde Pública, 38(2), 157-163.

Pitzner, J. K. \& Drummond, P. D. (1997). The Reliability and Validity of Empirically Scaled Measures of Psychological/ Verbal control and Physical/Sexual Abuse: Relationship between current negative mood and a history of abuse independent of other negative life events. Journal of Psychosomatic Research, 2, 125-142.

Rosenberg, M. (1989). Society and the Adolescent Self-Image. Princeton: Princeton University Press.

Sanders, B. \& Becker-Lausen, E. (1995). The measurement of Psychological Maltreatment: Early data on the Child Abuse and Trauma Scale. Child Abuse \& Neglect, 19(3), 315-323.

Seva, A., Sarasola, A., Merino, J. A. \& Magallon, R. (1992).Validity test of the GHQ-28 items in a subsample of young people. European Journal of Psychiatry, 6, 239-247.

Stewart, S. M., Lewinsohn, P. M., Lee, P. W., Ho, L. M., Kennard, B., Hughes, C. W., Emslie, G. (2002). Journal of Cross-Cultural Psychology, 33(6), 559-576.
Sukiennik, P. B. \& Salle, E. (2002). Depressão. Em M. C. O. Costa \& R. P. de Souza (Orgs.), Adolescência (pp. 340-349). Porto Alegre: Artmed.

Straus, M. A. (1979). Measuring familiar conflict and violence: The Conflict Tactics (CT) Scales. Journal of Marriage and the Family, 41, 75-88.

Trombeta, L. H. \& Guzzo, R. S. L. (2002) Enfrentando o cotidiano adverso: estudo sobre resiliência em adolescentes. Campinas: Alínea.

Ustárroz, L. F. (1997). Eventos estressantes, insatisfação na vida e morbidade psiquiátrica menor em Pelotas, RS. Dissertação de Mestrado, Universidade Federal de Pelotas, Pelotas.

Victora, C. G., Huttly, S. H., Fuchs, S. C. \& Olinto, M. T. A. (1997). The Role of Conceptual Frameworks in Epidemiological Analysis: A Hierarchical Approach. International Journal of Epidemiology, 26(1), 224-227.

Vitolo, Y. L. C., Fleitlich-Bilyk, B., Goodman, R. \& Bordin, I. A. S. (2005). Crenças e atitudes educativas dos pais e problemas de saúde mental em escolares. Revista Saúde Pública, 39(5), 716-724.

Wagnild, G. M. \& Young, H. M. (1993). Development and Psychometric Evaluation of Resilience Scale. Journal of Nursing Measurement, 1(2),165-178.
Recebido em 14.12.2005

Primeira decisão editorial em 22.02.2006

Versão final em 14.06.2006

Aceito em 07.06.2007 